\title{
Study between intravenous droperidol and granisetron as prophylactic antiemetic in prevention of PONV in gynaecological laparoscopic surgery
}

\author{
Amit Kumar
}

Assistant Professor, Department of Anesthesiology, M G M Medical College and L.S.K. Hospital, Kishanganj, Bihar India. Email: amit1982@gmail.com

\begin{abstract}
Background: Postoperative nausea and vomiting is one of the most common complications following general anaesthesia and surgery. It is one of the most bothersome adverse effects associated with surgery, as distressing as the pain associated with surgery. Method: Study was carried out in the department of Anaesthesiology, M.G.M. Medical College and L.S.K. Hospital during the period of January 2018 to September 2019 to determine the safety, efficacy and practicability of these two drugs as prophylactic antiemetic for prevention of Postoperative nausea and vomiting in adult female patients. 60 adult female patients with physical status of ASA grade I, II, scheduled for gynaecological laparoscopic surgery were randomly allocated to fall in three groups. Results: In this study we found that 11 cases of nausea and 09 cases of vomiting occurred in normal saline or placebo group. Where in droperidol group there were 4 cases of nausea and 5 cases of vomiting. In granisetron group there was 6 cases of nausea and 4 cases of vomiting. When calculated statistically it was found that the result was statistically significant when placebo group was compared with droperidol and granisetron group. But by using test of proportion (t- test) between droperidol and granisetron no statistically significant difference was found regarding Postoperative nausea and vomiting. Conclusion: Undergoing gynaecological laparoscopic surgery, both droperidol and granisetron were equally effective as prophylactic antiemetic in the prevention of Postoperative nausea and vomiting without any untoward side effects in the intraoperative and postoperative Time.

Key Word: droperidol, granisetron.
\end{abstract}

\begin{tabular}{l} 
*Address for Correspondence: \\
Dr. Amit Kumar, Assistant Professor, Department of Anesthesi \\
Email: amit1982@gamail.com \\
Received Date: $12 / 12 / 2019$ Revised Date: 06/01/2020 Accep \\
DOI: https://doi.org/10.26611/101513231 \\
\begin{tabular}{|l|l|}
\hline \multicolumn{2}{|c|}{ Access this article online } \\
\hline Quick Response Code: & Website: \\
\hline
\end{tabular} \\
\cline { 2 - 3 }
\end{tabular}

\section{INTRODUCTION}

Postoperative nausea and vomiting is one of the most common complications following general anaesthesia and surgery. It is one of the most bothersome adverse effects associated with surgery, as distressing as the pain associated with surgery. ${ }^{1}$ Minimizing patient morbidity and maximizing patient satisfaction are the important goal for health care providers. PONV is a complex condition that assumes greater importance as major mortality rating to surgery now decreases ${ }^{2}$. PONV costs have been estimated at $\$ 1.2$ billion a year in the United States alone ${ }^{3}$. In the "ether era" incidence of PONV reported was as high as $80 \%$. The replacement of older anaesthetic agent with shorter- acting and less emetogenic agent in conjunction with surgical refinements has reduced the overall incidence to $20 \%-30 \%$, which has been remarkably consistent over the past two decades ${ }^{4}$. Factors responsible for increased incidence of PONV are grossly divided into fixed factors and variable factors. Apfel et al. in 1999 found four highly predictive factors related to PONV. ${ }^{5}$ They are female gender, history of motion sickness or PONV, nonsmoker, and the use of perioperative opioids. Apfel CC et al. in 2002 found that if none, 1, 2, 3 or 4 of these risk factors were present then the incidence of PONV were $10 \%, 21 \%, 39 \%, 61 \%$ and $79 \%$ respectively. The 
simplified risk score was found favorable when compared with other predictive models 6 .

\section{METHODOLOGY}

Study was carried out in the department of Anaesthesiology, M.G.M. Medical College and L.S.K. Hospital during the period of January 2018 to September 2019 to determine the safety, efficacy and practicability of these two drugs as prophylactic antiemetic for prevention of Postoperative nausea and vomiting in adult female patients. 60 adult female patients with physical status of ASA grade I and II, scheduled for gynaecological laparoscopic surgery were randomly allocated to fall in any of the following three groups:

- GROUP- A will receive intravenous Normal Saline as placebo.

- GROUP- B will receive intravenous Droperidol $(25 \mathrm{mcg} / \mathrm{kg})$.

- GROUP- C will receive intravenous Granisetron $(40 \mathrm{mcg} / \mathrm{kg})$

The ages of the patients ranged from 21 to 38 years and body weight was 51 to $72 \mathrm{kgs}$. The patients were monitored intraoperatively from the beginning to the end of the operative procedure and postoperatively in terms of continuous ECG monitoring, pulse rate, $\mathrm{NIBP}, \mathrm{SPO}_{2}$, at an interval of 15 minutes. Postoperative assessment for occurrence of nausea, vomiting, recovery from anaesthetic effect, adequacy of ventilation and movement were carried out in the post anaesthesia care unit. The assessments were done first at $30 \mathrm{~min}$ after the arrival of the patient in the post anaesthesia care unit, then at 30 mins interval for upto 3 hours. Then at 6 hourly interval for upto 24 hours. Emesis score was assessed after asking the patients for any occurrence of nausea, retching, or vomiting. Recovery score was judged by noting the patient sedation status. It was done on the basis of seeing the spontaneous eye opening, response to verbal command and orientation to time, place and date of birth. Movement score was judged by the spontaneous and purposeful movement present or not or whether the patient can perform it on demand. During intraoperative period no patient developed significant hypoxia, hypercarbia, hypotension or hypertension. Hemodyanamic stability was maintained throughout the procedure in all three groups.

\section{RESULTS}

\begin{tabular}{cccc} 
Table 1: Mean and SD value of pulse rate ( beats/ min) In three groups (Intraoperative) \\
\cline { 2 - 5 } TIME INTERVAL & GROUP - A & GROUP - B & GROUP- C \\
\hline 0 min & $102.23 \pm 4.37$ & $101.09 \pm 4.37$ & $98.58 \pm 3.35$ \\
15 minutes & $107.0 \pm 3.46$ & $99.0 \pm 3.43$ & $101.21 \pm 3.42$ \\
30 minutes & $97.87 \pm 2.45$ & $98.91 \pm 3.93$ & $104.82 \pm 2.42$ \\
45 minutes & $97.23 \pm 4.56$ & $98.10 \pm 4.66$ & $98.09 \pm 5.23$ \\
60 minutes & $98.17 \pm 4.55$ & $98.27 \pm 5.57$ & $98.27 \pm 4.55$ \\
75 minutes & $96.89 \pm 1.33$ & $95.33 \pm 2.33$ & $96.23 \pm 4.66$ \\
90 minutes & $96.42 \pm 4.23$ & $97.34 \pm 5.45$ & $97.24 \pm 4.66$ \\
105 minutes & $100.58 \pm 6.23$ & $104.67 \pm 7.22$ & $99.25 \pm 6.74$ \\
\hline
\end{tabular}

showing pulse rate in three groups. The base line values were comparable in all three groups. Pulse rate was slightly decreased after 30 minutes in all groups. There was no statistical significance in these three groups.

\begin{tabular}{cccc}
\multicolumn{4}{c}{ Table 2: Mean and SD value of arterial blood pressure in three groups } \\
\hline TIME INTERVAL & GROUP - I & GROUP- II & GROUP -III \\
\hline 0 min & $98.71 \pm 4.31$ & $96.72 \pm 4.35$ & $98.71 \pm 3.34$ \\
15 minutes & $95.75 \pm 4.92$ & $95.47 \pm 4.93$ & $94.73 \pm 4.91$ \\
30 minutes & $90.12 \pm 6.51$ & $91.17 \pm 6.53$ & $90.17 \pm 6.52$ \\
45 minutes & $96.51 \pm 1.91$ & $95.50 \pm 1.92$ & $94.50 \pm 1.91$ \\
60 minutes & $97.71 \pm 4.35$ & $98.70 \pm 4.36$ & $98.71 \pm 4.33$ \\
75 minutes & $94.6 \pm 5.36$ & $97.30 \pm 6.52$ & $96.3 \pm 6.51$ \\
90 minutes & $94.3 \pm 4.37$ & $94.7 \pm 5.37$ & $95.8 \pm 5.36$ \\
105 minutes & $95.67 \pm 1.79$ & $94.6 \pm 1.82$ & $95.7 \pm 1.81$ \\
\hline
\end{tabular}

shows the changes in mean arterial blood pressure in three groups. The base line mean arterial blood pressure were comparable in three groups. At 15 minutes there was slight fall in blood pressure in all three groups. However, when compared statistically there was no significant difference in three groups at different time intervals.

TIME INTERVAL GROUP - I $\quad$ GROUP- II $\quad$ GROUP- III




\begin{tabular}{cccc}
\hline Omin & $99.26 \pm 1.47$ & $97.36 \pm 1.18$ & $97.76 \pm 1.39$ \\
15 minutes & $98.26 \pm 1.85$ & $99.04 \pm 0.64$ & $99.24 \pm 0.04$ \\
30 minutes & $98.23 \pm 0.28$ & $99.74 \pm 0.37$ & $99.92 \pm 0.92$ \\
45 minutes & $99.12 \pm 1.11$ & $99.50 \pm 0.09$ & $99.34 \pm 1.38$ \\
60 minutes & $99.81 \pm 0.56$ & $99.82 \pm 0.67$ & $98.82 \pm 0.55$ \\
75 minutes & $98.92 \pm 1.37$ & $99.76 \pm 1.28$ & $97.61 \pm 1.11$ \\
90 minutes & $98.48 \pm 0.46$ & $99.34 \pm 0.38$ & $98.42 \pm 0.34$ \\
105 minutes & $99.42 \pm 1.21$ & $99.56 \pm 1.38$ & $99.41 \pm 1.24$ \\
\hline
\end{tabular}

The intraoperative changes in $\overline{\mathrm{SPO} 2}$ in three groups. There was no statistically significant changes in preoperative value in three groups.

\begin{tabular}{ccc} 
Table 4: Comparison of emesis score in 3 groups in 24 hours \\
\hline Groups & Nausea and / or retching & Vomiting \\
\hline GROUP-A & 11 & 09 \\
GROUP-B & 04 & 05 \\
GROUP-C & 06 & 04 \\
\hline
\end{tabular}

Postoperative nausea and vomiting in all three groups, in 24 hours postoperative period. The test of proportion (z-test) shows that there is statistically significant difference $(\mathrm{P}<0.01)$ regarding nausea and retching among Group- A (placebo) and Group-B (Droperidol); and also among the placebo and (granisetron) Group- $\mathrm{C}(\mathrm{p}<0.05)$. But there was no significant statistical differences found among Group-B and group-C regarding nausea and/ or retching.

\section{DISCUSSION}

The high incidence of PONV after laparoscopic surgery gives rise to the question of antiemetic prophylaxis. It is evident from several studies that antiemetic prophylaxis is justified after laparoscopic surgery and an antiemetic should be given regularly, using standard drug and dose. Recently Scuderi et al. in his study raised a query regarding use of this antiemetic prophylaxis ${ }^{7}$. Fisher et al. also supported this findings ${ }^{8}$. According to them there appears to be little evidence to support routine prophylactic administration of antiemetics. But there are lots of works that clearly show increased patient satisfaction with prophylactic antiemetics, in contrast to placebo with rescue therapy in PACU for patients with symptoms in wait- andsee- approach ${ }^{9,10}$. Palazzo and Strunin in 1984 in a study concluded that now a days, the incidence of emetic problems associated with anaesthesia in the absence of antiemetic is still around $30 \%{ }^{11}$. In this placebo-controlled, prospective, randomized, clinical study the efficacy of two different groups of antiemetics was compared for preventing PONV following gynaecological laparoscopic surgery. The two study drugs were inj droperidol (butyrophenone group) and inj granisetron $\left(5 \mathrm{HT}_{3}\right.$ receptor antagonist). As PONV was being evaluated, so routine use of antiemetic was withheld as premedication. This current study was also carried out to find out the safety, efficacy and practicability of these two drugs in terms of PONV and to compare the recovery profile of patients in these groups (Group-A, Group-B, Group-C). 60 adult non-pregnant female patients aged $21-38$ years were enrolled in the study, belonging to ASA physical status grade I and II, scheduled for gynaecological laparoscopic surgery. The mean age was $29.45 \pm 1.30$ for placebo (group - A), 28.48 \pm 1.77 for droperidol (group $-\mathrm{B}$ ) and $28.47 \pm 2.33$ for granisetron (group - C) group. Patients of age group of 21 years to 38 years were chosen because, this age group was associated with highest incidence of PONV. The rate of PONV is controversial in younger age group and less in older patients ${ }^{[12]}$. Female patients were selected because, it was evident from the study that females are $2-4$ times more prone to PONV than males [13]. Non-pregnant patients were chosen because; pregnancy is a contraindication to laparoscopy. Furthermore, anaesthesia and surgical stress may have some adverse outcome over the foetus. Also the pregnancy may influence the rate of PONV than in the non-pregnant female because of the delayed gastric emptying ${ }^{14}$. The physical status of these patients selected for this study was of the grade of ASA I and ASA II, so that the effect of the disease process in the pharmacokinetics and pharmacodyanamics of the drugs as well as the effect on the assessment of PONV could be eliminated and also the adverse pathophysiological effects of laparoscopy might not be increased. Therefore, the patients who had the evidence of major cardiovascular, pulmonary, hepatic, renal, hematological, endocrinal or metabolic disorders were excluded from the study. The surgical procedure included diagnostic gynaecological laparoscopy mainly for infertility and pelvic inflammatory diseases, ovum retrieval surgery. Laparoscopic surgery was chosen because, apart from strabismus surgery, this surgery is regarded as the standard operation for the study of PONV, with gynaecological laparoscopic surgery still having the higher rates ${ }^{15}$. Preoperative visit before the day of surgery was conducted routinely to establish a good rapport with the patients. Patients were given light nonresidue diet on the night before surgery and kept on overnight fasting to clear the gut and stomach of food material. The patients were premedicated with tablet 
diazepam $5 \mathrm{mg}$ orally the night before and 90 minutes before arrival to operating room to achieve a desirable anxiolytic property, as anxiety has been suggested as a factor which can increase the incidence of PONV ${ }^{16}$. The patients were hydrated before anaesthesia with physiological solution like Ringer's lactate before induction of anaesthesia to replenish the loss during overnight fasting. This had also a favorable outcome on the hemodyanamic variables during the intraoperative period. Also it is evident from the study that preoperative hydration reduce the incidence of nausea and vomiting ${ }^{17}$. In our study in the postoperative period we found that 11 cases of nausea and 9 cases of vomiting in the placebo group who got normal saline, whereas in droperidol group there were 4 cases of nausea and 5 cases of vomiting and in granisetron group there were 6 cases of nausea and 4 cases of vomiting. When calculated statistically it was found that both droperidol and granisetron groups were superior in reducing nausea and vomiting than placebo group.

\section{CONCLUSION}

As prophylactic antiemetic in prevention of ponv in gynaecological laparoscopic surgery, both droperidol and granisetron were equally effective as prophylactic antiemetic in the prevention of Postoperative nausea and vomiting without any untoward side effects in the intraoperative and postoperative Time.

\section{REFERENCES}

1. Watcha MF, White PF. Postoperative nausea and vomiting , do they matter? Europian Journal of Anaesthesia 1995; 12(Suppl 10): 18 - 23.

2. David Cameron, Tong Joo Gan. Management of postoperative nausea and vomiting in ambulatory surgery . Anesthesiology. Clin N Am 2003; (21): 347 36.

3. Habib AS, Gann TJ. Combination therapy for Postoperative nausea and vomiting - a more effective prophylaxis? Ambul Sung 2001; 9: 59 - 71 .
4. Watcha MF, White PF . Postoperative nausea and vomiting its etiology, treatment and prevention. Anesthesiology 1992; $77: 162-84$.

5. Apfel CC, Laara E, Koivuranta M, et al. . A simplified risk score for predicting Postoperative nausea and vomiting. Anesthesiology 1999; 91: 693 - 700

6. Apfel CC, Krane P, Eberhart LHJ, et al.. Comparison of Predictive models for postoperative nausea and vomiting . Br. J Anaesth 2002; $88: 234-40$.

7. Scuderi PE, James RL, Harris L, et al.. Antiemetic prophylaxis does not improve outcomes after outpatient surgery when compared to symptomatic treatment. Anesthesiology 1999; 90: $360-71$.

8. Fisher DM. Surrogate outcomes : meaningful not [editorial]. Anesthesiology 1999; 90: 355 - 6 .

9. Sadasivam S, Shende D, Madem R. Prophylactic ondansetron in the prevention of postoperative nausea and vomiting following paediatric strabismus surgery : a dose response study. Anesthesiology 2000; 92: 1035 42.

10. Tang J, Watcha MF, White PF. A comparison of costs and efficacy of ondansetron and droperidol as prophylactic antiemetic therapy for elective outpatient gynaecologic procedures. Anesth Analg 1996; 83: $304-$ 13.

11. Palazzo MGA, Strumin L. Anaesthesia and emesis II. Prevention and management . Canadian Anaesthetists Society Journal 1984; $31: 407-15$

12. Vance J, Neill R, Nouis W. The incidence and aetiology of postoperative nausea and vomiting in a plastic surgical unit . Br. J Plastic Sung 1973; 26: 336 - 339.

13. Coher M, Duncan P, et al.. The post operative interview assessing risk factors for nausea and vomiting. Anesth Analg $1994 ; 78: 7-16$.

14. Brock - Utne JB, Dow TGB, Dimopoulus GE et al. Gastric and lower oesophageal sphincter pressure in early pregnancy. Br J Anaesth 1981; 53: 381 - 84.

15. Tay HS, Chin HH. Acid aspiration during laparoscopy. Anaesth Intens Care 1989; 6: 314.

16. Quinn A, Brown J, Wallace P, Asbury A . Studies in postoperative sequelae. Nausea and vomiting still a problem . Canadian Anaesthetists Society Journal 1976; $23: 466-69$

17. Bennett J, Mc Donald T, Licblich, et al.. Preoperative Rehydration in ambulatory anaesthesia for dentoalveolar surgery. Oral Med Oral Pathol Aral Radiol Endod 1999; $88: 279-284$.

Source of Support: None Declared Conflict of Interest: None Declared 